\title{
Luteinizing Hormone in Controlled Ovarian Stimulation
}

\author{
${ }^{1}$ Arveen Vohra, ${ }^{2}$ Kamini A Rao
}

\begin{abstract}
The role of follicle stimulating hormone (FSH) in assisted reproductive technology is well understood, though there is still no published consensus on the need for exogenous luteinizing hormone (LH) in controlled ovarian stimulation. There is a dilemma regarding the usefulness of LH supplementation in controlled ovarian stimulation despite growing understanding of the LH and FSH interrelation and their effects on fertilization and implantation. This review revisits the physiological role of $\mathrm{LH}, \mathrm{LH}$ receptors and the concept of $\mathrm{LH}$ therapeutic window. With the availability of LH activity from different sources, there is a need to understand the differences between recombinant human $\mathrm{LH}(\mathrm{r}-\mathrm{HLH})$, human menopausal gonadotropin and human chorionic gonadotropin ( $\mathrm{hCG}$ ). It has been observed that adjuvant $\mathrm{r}-\mathrm{HLH}$ provides precise control over the dose of $\mathrm{LH}$ bioactivity administered to target the therapeutic window. This review discusses about the various patient subgroups that may benefit from LH supplementation. The use of $\mathrm{r}-\mathrm{HLH}$ is recommended in women with poor response in a previous cycle or suboptimal follicular growth in an ongoing ovarian stimulation cycle by day 6 to 8 of stimulation. Exogenous LH administration should also be considered in women at risk of suboptimal response, specifically age $>35$ years and women treated with $\mathrm{GnRH}$ analogues (agonists or antagonists) during ovarian stimulation causing over suppression of endogenous $\mathrm{LH}$ and FSH pituitary secretion. Further research is needed to identify LH polymorphisms, adequate dosing, cost efficacy, need for rLH and hCG supplementation in different patient profiles for maximum benefit during controlled ovarian stimulating (COS).
\end{abstract}

Keywords: Luteinizing hormone, IVF, Ovarian stimulation, Recombinant LH, LH window, LH deficiency.

How to cite this article: Vohra A, Rao KA. Luteinizing Hormone in Controlled Ovarian Stimulation. Int J Infertil Fetal Med 2014; 5(3):75-86.

Source of support: Nil

Conflict of interest: None

Date of Received: 28-11-14

Date of Acceptance: 10-12-14

Date of Publication: September 2014

\section{INTRODUCTION}

The anterior pituitary secretes the major hormone releasing factors-follicle stimulating hormone (FSH), luteinizing hormone (LH), thyroid-stimulating hormone (TSH),

\footnotetext{
${ }^{1}$ Consultant, ${ }^{2}$ Medical Director

1,2 Department of Reproductive Medicine, Milann-The Fertility Centre, Bengaluru, Karnataka, India
}

Corresponding Author: Arveen Vohra, Consultant, Department of Obstetrics and Gynecology, Milann-The Fertility Centre Bengaluru, Karnataka, India, e-mail: dr.arveen@gmail.com and adrenocorticotropic hormone (ACTH) along with growth hormone (GH) and prolactin. Follicle stimulating hormone and LH have markedly different functions in the control of ovarian physiology though secreted from common cells. The control of FSH and LH is through a combination of differential control of their synthesis and secretion by gonadotropin-releasing hormone (GnRH).

As an oocyte matures within a follicle, a myriad of complex hormonal events take place, particularly during the follicular phase. The two gonadotropins LH and FSH play distinct but complementary roles in ensuring follicular growth and ovulation. These two gonadotropins are active in the final weeks of the development of a mature surviving oocyte, with initiation of folliculogenesis occurring independently of gonadotropic stimulation. Secretion of LH and FSH in an orderly fashion is therefore crucial to the final development of a mature oocyte.

Although, recent studies have facilitated better understanding of the LH and FSH hormone interrelation and its effect on fertilization and implantation, there is still confusion on the usefulness of LH supplementation in controlled ovarian stimulation. Therefore, the use of recombinant human LH in controlled ovarian hyperstimulation $(\mathrm{COH})$ should be guided by a rationale that is based on the individualized patient profile and requirements. The concept of an LH therapeutic window has implications for the choice of LH supplementation in assisted reproduction technology. Endogenous LH production is pulsatile and occurs in response to the pulsatile release of GnRH from the hypothalamus. The most physiological way to maintain LH concentrations is to utilize endogenous LH secretion.

\section{PHYSIOLOGICAL ROLE OF LUTEINIZING HORMONE}

Follicle stimulating hormone and LH both are glycoproteins that share identical $\alpha$ subunits along with TSH and placental human chorionic gonadotropin (hCG) differing only in the structure of their $\beta$ subunits, which confers receptor specificity. Luteinizing hormone is required for the growth of preovulatory follicles, ovulation and luteinization of the dominant follicle. The LH receptor luteinizing hormone receptor (LHR) gene is expressed in granulosa cells of human antral follicles throughout the follicular phase and is significantly associated with expression of the CYP19a1 gene and with the corresponding 


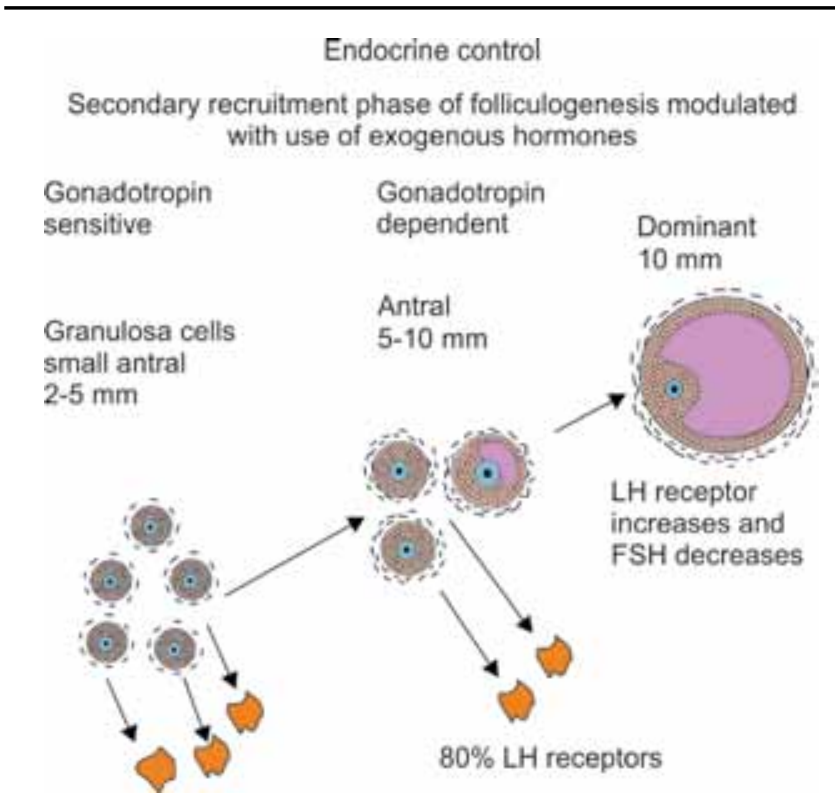

Fig. 1: Luteinizing hormone receptors in different stages of folliculogenesis

follicular fluid concentrations of estradiol and progesterone. Luteinizing hormone receptor gene is expressed in human granulosa cells from around a diameter of 5 to $6 \mathrm{~mm}$ and until ovulation. Luteinizing hormone receptor expression peaks in granulosa cells just before initiation of the midcycle of gonadotropins and may affect human follicular development earlier than previously believed. In small antral follicles with a diameter of 3 to $10 \mathrm{~mm}$, around $80 \%$ express LHR; exhibiting strong positive associations to estradiol, progesterone, $\mathrm{AMH}$, and inhibin-B in the corresponding follicular fluid, suggesting that functional receptors are present on the granulosa cells ${ }^{1}$ (Fig. 1).

The FSH receptors are present over the granulosa cells. The ovarian theca and granulosa cells are the principal sites of LH bioactivity, although LH receptors are also present in extragonadal sites, such as the uterus. ${ }^{2}$ During the follicular phase of the menstrual cycle, LH induces androgen synthesis by theca cells that is essential for robust steroidogenesis; stimulates proliferation, differentiation, and secretion of follicular theca cells and also increases LH receptors on granulosa cells. The preovulatory LH surge drives the oocyte into the first meiotic division and initiates luteinization of theca and granulosa cells. The resulting corpus luteum produces high levels of progesterone and some estrogen.

\section{THERAPEUTIC LH WINDOW}

Luteinizing hormone in association with FSH is recommended for stimulating follicular development in women with severe LH and FSH deficiency with an endogenous serum LH concentration $<1.2 \mathrm{IU} / 1$. According to the threshold theory of LH in ovarian function, the ovarian follicle requires a minimal amount of LH for steroidogenesis $(<1 \%$ of receptors attached by $\mathrm{LH})$. There is also a ceiling beyond which excessively high concentrations of LH may actually suppress granulosa aromatase activity and inhibit cell growth. The LH ceiling is dependent on timing of the menstrual cycle but for optimal follicle development, this concentration is typically between $1.2 \mathrm{IU} / 1$ and $5 \mathrm{IU} / \mathrm{l}^{3}$

The LH dependent phase of preovulatory follicular development proceeds normally only if $\mathrm{LH}$ is present at concentrations within the LH window, which is above the threshold level and beneath the ceiling value. The developing follicles have specific requirements for exposure to LH beyond which normal maturation ceases which gave rise to the concept of LH ceiling, meaning that each follicle would have an upper limit of stimulation. The LH ceiling may be higher in larger follicles and lower in smaller ones. As a consequence, an increasing LH concentration would promote leading follicle progression (being below its ceiling) and degeneration of secondary ones (by overcoming their ceiling). A dynamic interplay between LH secretion and receptor expression by different ovarian compartments governs the selection of dominant follicles. The small follicles in granulosa cells do not express LH receptors so LH may indirectly promote their degeneration. It should be recognized that the LH threshold evolves through the follicular cycle
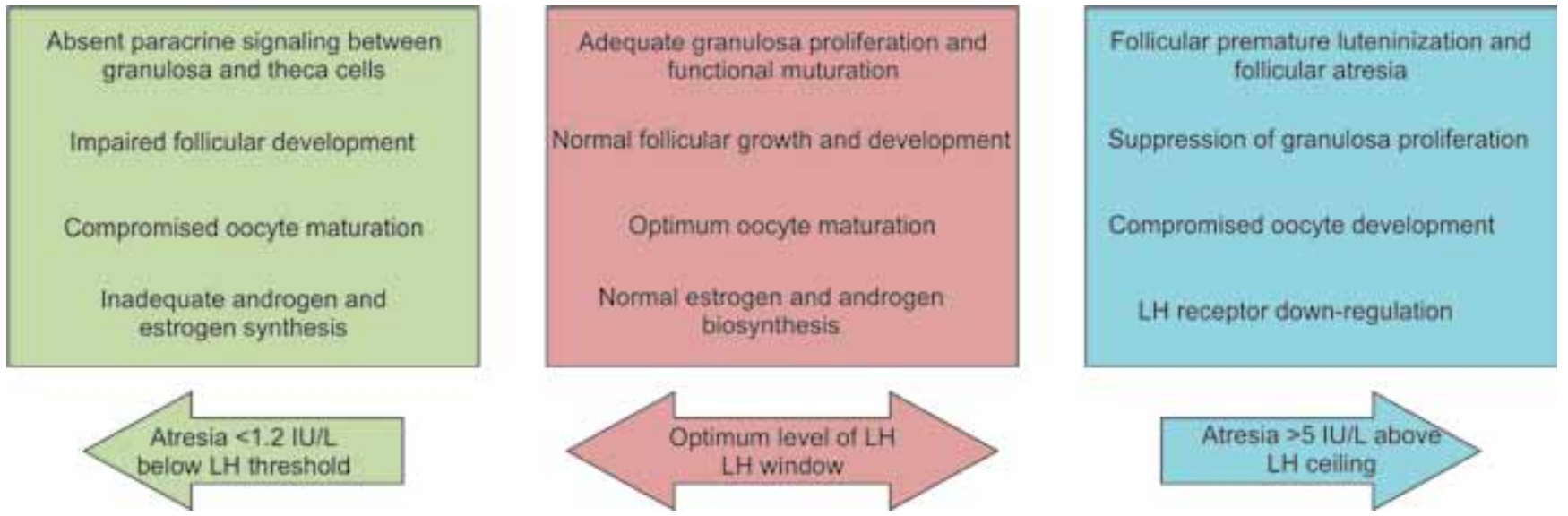

Fig. 2: Impact of different concentrations of luteinizing hormone on the menstrual cycle 
and conceptually should become higher as the follicles grow $^{4}$ (Fig. 2).

The usefulness of exogenous LH in ovarian stimulation protocols in assisted reproduction continues to be controversial. Though there are no clear guidelines on when exactly LH needs to be added. It is critical that add-back LH is administered in appropriate patients as excess of LH can cause suppression of granulosa cells and follicular atresia.

\section{TWO-CELL, TWO-GONADOTROPIN CONCEPT}

Follicle stimulating hormone is essential for follicular recruitment and development, as well as for inducing many enzymes and hormones (e.g. aromatase, inhibin) that are subsequently controlled by LH and required for continued follicle maturation. ${ }^{5} \mathrm{LH}$ stimulates adenylate cyclase and cyclic AMP production resulting in mitochondrial cholesterol transport and steroidogenesis, essential events leading to oocyte maturation and ovulation. ${ }^{6}$ Not only does LH play an important function in the periovulatory and ovulatory events within the ovary, but also LH receptors have been identified in the human endometrium, raising the possibility that LH has a necessary function in implantation. ${ }^{7}$

Luteinizing hormone seems to play two roles during folliculogenesis. One is exerted in the theca compartment and consists of induction of androgen production. The second begins during the intermediate follicular phase, involves granulosa cells, and consists of inducing the local production of various molecules. These factors promote the growth of granulosa cells, which in turn regulate oocyte maturation. These two mechanisms are closely related and probably support each other. According to the so-called 'spare receptor hypothesis', at a time when inhibin B and IGF-1 are adequately secreted, androgen synthesis and release are optimal even with $<1 \%$ of $\mathrm{LH}$ receptors occupied. ${ }^{3}$

Luteinizing hormone optimizes final stages of maturation. FSH stimulated estrogen production from

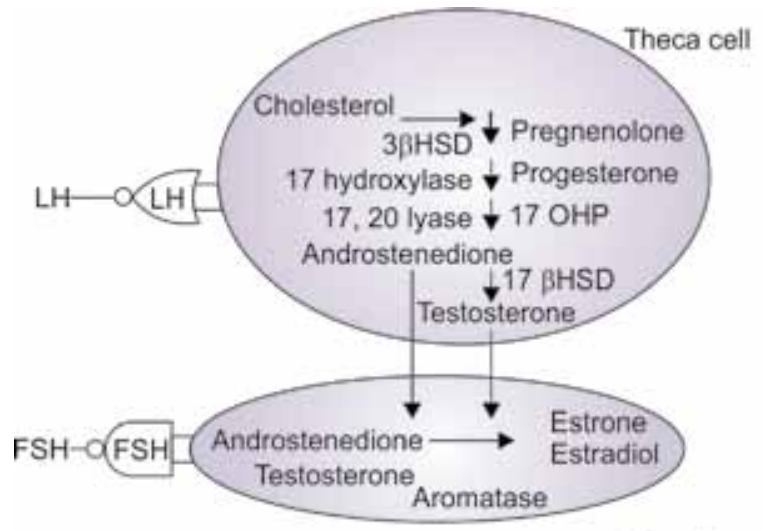

Granulosa cel

Fig. 3: Two cell, two gonadotropin theory the granulosa cells is dependent on LH stimulated androgen production by the theca cells. This is not fully functional till later part of antral development. Theca cells are characterized by expression of LH receptors, $3 \beta$ hydroxy steroid dehydrogenase and cyp-P450c 17 essential for internalizing LDL cholesterol into mitochondria and thereby converting 21 carbons to androgens. The steroidogenic acute regulatory protein (StAR protein) is the primary regulator of production of androstenedione, which subsequently diffuses into granulosa cells to serve as an estrogen precursor. In the preovulatory follicle, cholesterol in theca cells arises from circulating lipoproteins and de novo biosynthesis. ${ }^{8}$

Granulosa cells express FSH receptors and cyp-450 aromatase to convert androgen substrate from theca into estrogens. Increased expression of aromatase indicates increased follicular maturity. Exclusive function of P450c 17 in theca cells and aromatase in granulosa cells is the basic principle of 2-cell 2-gonadotropin theory.

Pure FSH treatment causes early development of follicle, but estradiol production is limited. Some aromatization occurs from the androgens of adrenal glands, but robust steroidogenesis is not possible. Only dominant follicle with more FSH receptors, high aromatase activity and robust steroidogenesis is selected as dominant follicle. Following the mid-cycle LH surge, granulosa cell mitosis is blocked, and oocyte meiosis is resumed (Fig. 3).

The best evidence for a critical role of LH in ovulation induction has come from studies evaluating pregnancy outcomes in women with hypogonadotropic hypogonadism (WHO class I). In the presence of low endogenous LH production, particularly among women with LH concentrations $<1.2 \mathrm{mIU} / \mathrm{ml}$, studies utilizing FSH alone for ovulation induction have found poor pregnancy outcomes. ${ }^{9}$ Consequently, both FSH and LH supplementation appear to be critical for optimal follicular development, implantation and pregnancy. At the other extreme, elevated LH concentrations have likewise been associated with poor fertilization, poor implantation and detrimental effects on pregnancy rates. While insufficient LH results in inadequate steroidogenesis, excessive LH may suppress aromatase activity and inhibit cell growth. Taken together, this evidence supports the theory that a therapeutic window exists for $\mathrm{LH}$, above or below which maximum reproductive outcomes may be negatively impacted. ${ }^{6}$

Luteinizing hormone administration may induce atresia of smaller follicles and the remaining larger follicles may represent those with greater reproductive competence. Improved endometrial receptivity may also be produced as implantation rates have been shown to positively correlate with increasing doses of $\mathrm{LH}^{10}$

Nevertheless, whether the LH component of human menopausal gonadotropins (HMG) for ovarian stimulation 
is advantageous, disadvantageous or inconsequential to the ultimate outcome of interest, pregnancy rates, has been a matter of controversy.

\section{LUTEINIZING HORMONE: DIFFERENT FORMS}

It is clear that there is no absolute requirement for $\mathrm{LH}$ in the exogenous gonadotropin used for ovarian stimulation, but it has potential advantages in definable situations. Until recently, the only available source of exogenous LH activity has been HMG, a urine-derived preparation containing both $\mathrm{FSH}$ and $\mathrm{LH}$, which comprises about $5 \%$ of the total protein content. Luteinizing Hormone activity can currently be derived from HMG preparations, urinary hCG, recombinant LH and recombinant hCG.

\section{Role of Human Menopausal Gonadotropins}

Human menopausal gonadotropins are urinary-derived gonadotropins containing roughly equivalent amounts of LH and FSH bioactivity (approximately 75 IU of each). For many years, HMG was the only gonadotropin available. Despite their widespread use, there have been several proposed disadvantages of these compounds, including protein contamination leading to local allergic reactions and batch-to-batch inconsistencies, as well as supply limitations. Despite significant improvements in processing, different HMG preparations are subject to wide variation in LH quantity and bioactivity and with increased purification, more LH is lost. Thus, hCG is often added in an attempt to boost the LH bioactivity to meet the required LH activity range (FSH:LH ratio = 1:1) as stated in the pharmacopoeia. This may result in the end product having much more hCG than LH activity.

The Van Hell bioassay of HMG only detects LH bioactivity and does not distinguish LH from hCG. Analysis of one HMG product (Menopur) showed that the content of hCG was more than 10 times higher than LH. Another analysis also showed that about $95 \%$ of the $\mathrm{LH}$ receptor bioactivity in one HMG product (Menopur) was attributed to hCG, with less than $5 \%$ contributed by pure $\mathrm{LH}^{11}$

Owing to the controversial nature of the topic, in the past decade, five meta-analyses have contributed to the current understanding of the effect of HMG compared with FSH-only protocols on assisted reproduction outcomes. $^{12,13}$

With regard to the type of pituitary down-regulation, data showing that HMG cycles yield a 3 to $4 \%$ higher live birth rate than $\mathrm{rFSH}$-only cycles have come from trials utilizing long GnRH agonists. ${ }^{13}$ In a single randomized controlled trial comparing HMG to $\mathrm{rFSH}$ while using a GnRH antagonist, Bosch et al demonstrated a 3\% higher live birth rate in the HMG group. However, this finding was not significant as even though the similar increase in live birth rates is notable, the data on HMG use in GnRH antagonist cycles is too limited to make definitive conclusions. ${ }^{14}$

The studies have estimated that HMG protocols may provide a 3 to $4 \%$ but significant improvement in live birth rate compared with rFSH-only protocols when a long GnRH agonist protocol was utilized. While statistically significant, the clinical significance of this finding remains controversial. An important patient consideration has been that significantly fewer ampoules of gonadotropins were required among those receiving HMG as compared with rFSH-only protocols, resulting in a lower cost per live birth in the HMG group without compromising patient safety. With the potential for lower treatment costs, there seems to be a benefit to the inclusion of HMG in treatment protocols. ${ }^{15}$

\section{Role of Recombinant Human LH}

Recombinant human FSH (r-HFSH) replacement has been used in ovarian stimulation protocols since its approval in 1994. There is increasing interest in exploring the role of LH and its optimal use in relation to assisted reproduction. With the availability of recombinant LH (rLH), stimulation protocols have incorporated its use in place of HMG. Pharmacodynamic studies of rLH have shown it to have a similar volume of distribution, half-life and bioactivity to urinary products. These profiles have not differed whether rLH was administered sc or im and have not been shown to affect FSH pharmacodynamics when coadministered. Since LH has a precise therapeutic window, the physician needs to have precise control over the activity of exogenous LH administered. rLH is analogous to endogenous LH and characterized by high purity, precision of dosing and consistency. When administered by subcutaneous injection, rLH has a terminal half-life of 24 hours. rLH is structurally and functionally analogous to endogenous human LH. In a RCT with patients with a suboptimal response to stimulation with a long GnRH agonist stimulation protocol that compared adding higher doses of $\mathrm{rFH}$ vs adding rLH or HMG, those given rLH had higher live-birth rates (40.7\%) than those given HMG (18\%). The rLH group also had higher implantation rates. The authors speculated that the differences between the two preparations could produce different biological effects in women who exhibit a suboptimal response to FSH and concluded that using rLH is justified if better outcomes can be achieved., ${ }^{9,16,17}$

While some advocate add-back LH to the mid-follicular phase in ovarian stimulation cycles, others deem add-back LH to be unnecessary, justifying that the small amount of LH present after down regulation is enough 
to sustain theca and granulosa cell stimulation. ${ }^{18}$ However, supplementation with rLH has shown lower levels of cumulous cell apoptosis than treatment with FSH alone, possibly indicating improved oocyte quality in LH-supplemented cycles. The decreased granulosa cell apoptosis in the rLH group might be the result of lower levels of follicular fluid vascular endothelial growth factor; marker of maturity and quality of oocytes that is produced by granulosa and theca cells in response to FSH, LH, hCG and proliferative and apoptotic factors. ${ }^{19,} 20$

The importance of LH supplementation in studies of ovulation induction has been shrouded by conflicting data. The best evidence for a critical role of LH in ovulation induction has come from studies evaluating pregnancy outcomes in women with hypogonadotropic hypogonadism. In the presence of low endogenous LH production, particularly among women with LH concentrations $<1.2 \mathrm{mIU} / \mathrm{ml}$, studies utilizing $\mathrm{FSH}$ alone for ovulation induction have found poor pregnancy outcomes. ${ }^{9}$ Consequently, both FSH and LH supplementation appear to be critical for optimal follicular development, implantation and pregnancy. ${ }^{21}$

On the other hand, elevated LH concentrations have been associated with poor fertilization, poor implantation and detrimental effects on pregnancy rates. While insufficient $\mathrm{LH}$ results in inadequate steroidogenesis, excessive LH may suppress aromatase activity and inhibit cell growth. Taken together, this evidence supports the theory that a therapeutic window exists for LH, above or below which maximum reproductive outcomes may be negatively impacted. ${ }^{6}$

The larger randomized controlled trials have been published on the use of recombinant LH supplementation from day 6 in long agonist protocols and recombinant LH supplementation from day 1 of stimulation in antagonist protocols. These trials have failed to document that serum LH concentrations during stimulation or the addition of recombinant $\mathrm{LH}$ have overall clinical benefits in terms of higher pregnancy rates. However, LH concentrations were related to endpoints like duration of stimulation, gonadotropin consumption, number of oocytes retrieved, estradiol and preovulatory progesterone rises in many studies. This suggests many highly significant associations but no association with the key endpointpregnancy. The overall conclusion is that, irrespective of protocols, circulating LH concentration is a predictor of various important parameters. Data suggest that like HMG, rLH results in higher estradiol concentrations and decreased usage of $\mathrm{rFSH}$, but it does not demonstrate convincing evidence that $\mathrm{LH}$ improves pregnancy outcomes over rFSH alone. $^{14,22}$

There are several possible explanations for why there may be demonstrable evidence of benefit with HMG but not when utilizing rLH. First, the studies performed to date utilizing rLH have had much smaller patient populations. Secondly, the studies evaluating rLH represented a much more heterogeneous group than those evaluating HMG. Thirdly, rLH and HMG may differ in their biological activity due to differences in LH glycosylation patterns and because of the addition of hCG in HMG. Finally, because of the fixed ratio of LH:FSH found in HMG, any addition of LH is coincident to the addition of FSH. Finally, due to its cost, studies have estimated that rLH may add $45 \%$ to the cost of ovarian stimulation despite the reduction in $\mathrm{rFSH}$ use.

\section{Role of hCG in the Mid-to-Late Follicular Phase}

Luteinizing hormone and hCG are structurally quite similar and bind to the same receptors. However, their beta subunits are different. The two hormones differ in the composition of their carbohydrate moieties that, in turn, affects bioactivity and half-life. Except for the CTP on the beta-subunit of hCG (amino acids 113-145), the two hormones share more than $85 \%$ sequence homology. Relative to HLH, the serum half-life of hCG is more than twofold greater. hCG is more stable with slower plasma metabolic clearance rate. hCG has a higher binding affinity to the LH receptor, with 1 IU hCG having biological activity approximately 6 to 8 times greater than 1 IU LH. After subcutaneous injection, hCG exhibits a longer serum half-life, thus leading to the possibility of significant accumulation over time. ${ }^{10}$

\section{hCG/HMG may Induce LH Receptor Internalization}

A recent study comparing stimulation with HMG vs r-HFSH in women undergoing IVF/intracytoplasmic sperm injection treatment found statistically significantly reduced expression of $\mathrm{LH}$ receptor messenger RNA in ovarian granulosa cells in the HMG group, which was associated with altered expression of genes and proteins involved in steroidogenesis in preovulatory granulosa cells. $^{23}$

These studies show that hCG is not equivalent to $\mathrm{LH}$ and there are effects at the level of LH receptor internalization, which may explain why there is tolerance' or a lack of effect. Finally, it is known that there is a LH ceiling associated with the use of rLH. Hugues et al established that in World Health Organization type II anovulatory women undergoing follicular stimulation, this LH ceiling was $>60 \mathrm{mcg}$ or $1325 \mathrm{IU}$ rLH/day when these women showed atresia of non-dominant follicles, elevated progesterone concentrations and reduced clinical pregnancy rates. ${ }^{24}$

The role of hCG as a supplement to FSH in the late follicular phase-in long and short agonist and antagonist 
protocols has been reviewed in a recent meta-analysis of nine studies. From the four treatment strategies defined in the analysis, hCG supplementation in the GnRH antagonist group was associated with a nonsignificant benefit in clinical pregnancy rate. A pilot study which compared the effect of hCG supplementation exclusively in GnRH antagonist cycles, found comparable rates of oocyte yield and pregnancy in the hCG and control groups, but a reduction in FSH consumption in the former. Four randomized trials demonstrated that the use of hCG in the mid-follicular phase could significantly reduce FSH dosage without a reduction in pregnancy rates and may represent a decrease in stimulation-associated costs. However, these studies were not powered to detect pregnancy or live birth rates as primary endpoints. ${ }^{18}$

The current evidence suggests that hCG or LH in the mid-to-late follicular phase can support continued follicular progression and estradiol production in the face of partial or complete FSH withdrawal. The potential advantage of this protocol is the consumption of less FSH for ovarian stimulation, potentially leading to a cost saving. However, convincing cost analyses of mid-cycle hCG protocols are lacking. The data show mid-cycle hCG to be equivalent to FSH protocols and no advantages to outcomes have been reported. Before widespread use, larger studies are needed to evaluate pregnancy and live birth rates. ${ }^{25}$

\section{ROLE OF LH IN ASSISTED REPRODUCTIVE TECHNOLOGY}

\section{Exogenous LH and ART Outcome in Unselected Population}

There is no evidence that supplementation with LH activity is necessary in an unselected IVF population, it may yet be beneficial in select subgroups of patients who usually need high doses of FSH.

A meta-analysis of four randomized controlled trials comparing r-hFSH and HMG treatment in normogonadotropic patients undergoing a GnRH-a long protocol failed to show any statistically significant difference in the ongoing pregnancy rates and live birth rate. In a metaanalysis utilizing GnRH antagonist, an increase in peak estradiol concentrations and a higher number of mature oocytes but no benefit in implantation and pregnancy rates is noted. ${ }^{26}$

Another meta-analysis comparing r-HFSH vs r-HFSH plus rLH ovarian stimulation showed that for unselected patients undergoing assisted reproduction technology there is no difference between the two treatments in live birth rate and does not recommend adding $\mathrm{LH}$ to unselected patients (age $<35$ years). However, the authors mentioned that their conclusion should be interpreted with caution, as it was not statistically significant. ${ }^{27}$

Hence, the present evidence is insufficient to prove that LH administration in unselected groups is associated with any significant improvement in the IVF/ICSI outcome parameters like implantation and pregnancy rates, when compared with rFSH only stimulation. ${ }^{18}$

\section{EXOGENOUS LH AND ART OUTCOME IN SELECTED POPULATION}

\section{Severe Endogenous LH Deficiency}

Luteinizing hormone polymorphism contributes to severe endogenous LH deficiency. The LH receptor gene is known to carry as many as 282 single-nucleotide polymorphisms (SNPs). A common genetic LH $\beta$ variant $(\mathrm{v}-\beta \mathrm{LH})$ owing to the alterations in two polymorphic base changes in the $\beta$ subunit gene leading to changes in the amino acid sequence, Trp8Arg and Ile15Thr has been identified. It was earlier thought to be an immunological anomalous LH form. ${ }^{28}$

The shorter half-life of $\mathrm{v}-\beta \mathrm{LH}$ may be linked to the presence of extra glycosylation signal into the $\beta$ subunit that could lead to an addition of second oligosaccharide to Asn13 of the $\beta$ protein. It has been found that there is more potency of the overall LH activity of $\mathrm{v}-\beta \mathrm{LH}$ at the receptor site; however, its duration is shorter in vivo. Previous clinical trials conducted to determine the impact of this variant on reproductive health reported its association with ovulatory disorders, premature ovarian failure, hyperprolactinemia, luteal insufficiency, menstrual disorders, endometriosis and infertility. A few studies have noted low responseinsomewomenfollowing ovarianstimulation, resulting in a greater need for $\mathrm{r}-\mathrm{hFSH}$ (>2,500 IU) due to the presence of $\mathrm{v}-\beta \mathrm{LH}$. Based on the findings, the researchers suggest the potential of $\mathrm{v}-\beta \mathrm{LH}$ as a marker of ovarian responsiveness to $\mathrm{r}-\mathrm{hFSH}$. This role of $\mathrm{v}-\beta \mathrm{LH}$, if validated with further research, could facilitate clinicians in identifying patients requiring exogenous LH addition during ovarian stimulation. ${ }^{29}$

Hypogonadotropic hypogonadism (WHO class I) impairs pituitary neuroendocrine function that results in abnormally low LH and FSH concentrations. Such patients typically have amenorrhea with small ovaries having follicles in arrested development due to the lack of effective hypothalamic-pituitary activity. These women do not have sufficient endogenous LH for optimal follicular growth and steroidogenesis when treated with FSH alone and will typically benefit from FSH and LH for optimal follicular development.

Hypophysectomized animal studies and studies in hypogonadotropic hypogonadism women confirm that 
r-HFSH increases follicular growth in a dose-dependent manner, but is ineffective in stimulating synthesis of estradiol due to the extremely low or near-undetectable endogenous LH concentrations. The role of LH in hypophysectomized women and in women with isolated gonadotropin deficiency has been reviewed and concluded that although r-HFSH increases the growth of multiple ovarian follicles, serum LH, androstenedione and estradiol concentrations remain low or show only negligible increase. ${ }^{6,16,21}$

The reviews confirm that $\mathrm{LH}$ is physiologically essential for estradiol synthesis and that replacement to above the minimal threshold concentration is necessary in women with inherent gonadotropin insufficiency.

\section{Severe LH Deficiency due to Suppression by GnRH Analogs in Assisted Reproduction Technology}

Women treated with GnRH analogs (agonists or antagonists) during ovarian stimulation in IVF, may similarly experience severely reduced LH and FSH concentrations due to over suppression of endogenous LH and FSH pituitary secretion. In some patients, the suppressed baseline LH concentrations may reach levels seen in hypogonadotropic hypogonadism women (i.e. < 1.2 IU/1). However, not every patient undergoing ovarian stimulation requires exogenous LH replacement, as endogenous production of small amounts of LH may remain sufficient for theca cell function. The studies show poorer outcomes among those patients who have a lower LH concentration or a sharper fall in LH from baseline concentrations and in patients whose endogenous $\mathrm{LH}$ is low after GnRH agonist treatment. ${ }^{30}$

A randomized trial in oocyte donors that compared r-HFSH vs r-HFSH combined with rLH in an antagonist protocol showed a significantly higher metaphase-II oocyte count, fertilization rates, grade 1 embryos and implantation rates, in recipients whose embryos originated from donors receiving added $\mathrm{rLH}$ than in donors receiving GnRH antagonist and r-HFSH alone. Future research is required to lend further support to the use of adjuvant rLH in patients on antagonist ovarian stimulation protocols. ${ }^{31}$

There may be a role for other biomarkers, e.g. LH polymorphisms, such as the LH beta variant in further identifying LH-deficient patients. ${ }^{28}$ Another way to identify the severely LH-deficient patient is to look at the ovarian response to FSH stimulation. Supplementation of LH may benefit selected women with LH deficiency and suboptimal ovarian response during assisted reproduction technology as measured by clinical end-points, such as estradiol concentrations and follicular development.
The evidence for addition of $\mathrm{rLH}$ to $\mathrm{rFSH}$ in antagonist protocols for ovarian stimulation is still being accumulated and more research is needed.

However, we must remember that early overexposure of LH in ovarian stimulation can result in premature follicle luteinization of small follicles and follicular atresia leading either to cycle cancellation due to follicle maturation arrest or to poor-quality oocytes, all of which translates into severely compromised outcomes.

\section{POOR RESPONDERS}

Poor ovarian reserve is estimated to occur in around $26 \%$ of the ART procedures. Evidence indicates that $\mathrm{rLH}$ and $\mathrm{r}-\mathrm{hFSH}$ coadministration in these patients may help in improving ongoing pregnancy rates in poor responders and women of advanced age. ${ }^{14,20,32}$

On the contrary, some recent studies concluded that there is an insufficient evidence to validate the effectiveness of rLH in subjects with poor response undergoing ART. $^{33}$

Some clinicians use a short GnRH agonist or microflare protocol in some of their older or poor-responder patients. There is substantial evidence of a benefit of adding rLH to women who have a poor response to ovarian stimulation, including poor response in a previous cycle; and a suboptimal ovarian response with suboptimal follicular progression in a current cycle by days 6 to $8 .^{20}$

\section{PATIENTS AT RISK OF HYPORESPONSE DURING OVARIAN STIMULATION}

In an ongoing $\mathrm{COH}$ cycle, there is a group of patients who, after using suppressive GnRH analogs (either agonists or antagonists), develop severe LH deficiency and exhibit a suboptimal ovarian follicular response by days 6 to 8 . A review defines this suboptimal ovarian response as: (i) having no follicle $>10 \mathrm{~mm}$ by day 6; (ii) low estradiol concentration $<200 \mathrm{pg} / \mathrm{ml}$ by days 6 ; and (iii) poor progression or slowing of follicle growth, i.e. previously 1 to $2 \mathrm{~mm}$ progression/day slowing to less than $2 \mathrm{~mm}$ in 3 days. There is an opportunity in this group of patients to salvage the ongoing cycle through rLH supplementation. When compared with increasing r-HFSH dose, adding rLH on days 8 was associated with a better cumulative implantation rate (14.2 vs 10.5) and cumulative pregnancy rate (37.2 vs 29.3). ${ }^{20,34,35}$

There are recommendations for the use of LH supplementation in the ongoing cycle for patients with a history of prior poor response and patients who exhibit a suboptimal response during long agonist ovarian stimulation protocols. There is a possibility to optimize the ovarian response in both these patient groups 
through the addition of rLH. ${ }^{20,34}$ Thus, LH supplementation seems appropriate for poor responders where it restored the follicular and endometrial milieu and improves the cycle outcome.

\section{ADVANCED REPRODUCTIVE AGE}

A recent systemic review and meta-analysis concluded that the inclusion of rLH to FSH stimulation enhanced the clinical pregnancy and implantation rates in ART cycles in patients aged greater than or equal to 35 years. Similar results were reported in many other randomized trials. Similarly, a Cochrane review reiterated the usefulness of rLH in poor responders and advanced aged women at risk of spontaneous miscarriage. ${ }^{14,15,30,32}$

A retrospective observational study evaluating ART patients undergoing stimulation with an antagonist procedure reported clinical pregnancy success of $36 \%$ for patients aged more than 38 years treated with r-hFSH and rLH compared with $19.1 \%$ for those stimulated with r-hFSH and HMG. An open-label randomized controlled study found that $\mathrm{rLH}$ is beneficial in improving the implantation rate in women aged 36 to 39 years, but not so in those younger than 36 years of age. This might be due to the fact that the serum androgen levels decline steeply with age, as does the response to FSH stimulation. Change in LH receptors of theca cells with age renders ovaries less sensitive to LH. Hence, exogenous LH administration enhances follicular androgen production followed by its aromatization to estrogen. It also controls progesterone production by granulosa cells, which is also FSH dependent (Fig. 4).

Based on these clinical studies and personal clinical experience, starting adjuvant rLH on either day 1 of stimulation or days 6 to 8 may be beneficial in patients older than 35 years in long agonist or antagonist protocol ovarian stimulation. Monitoring of the response

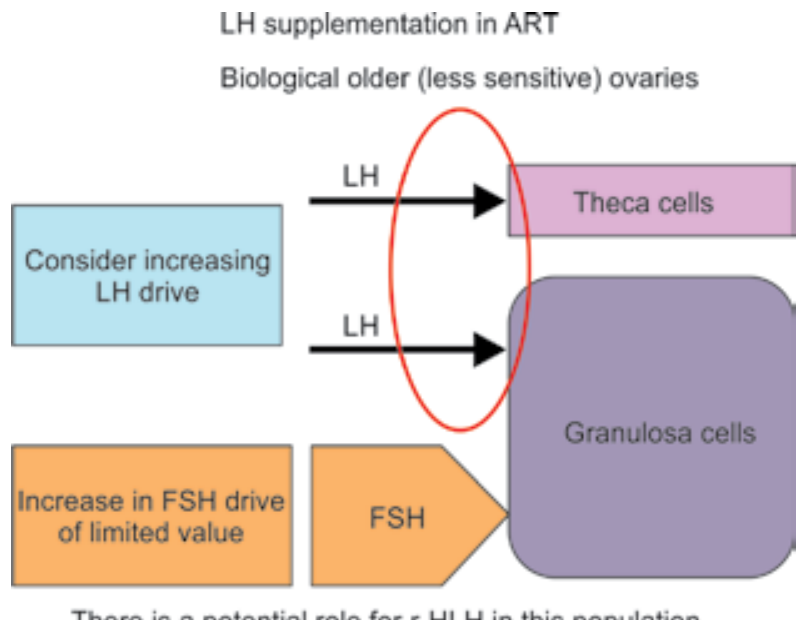

There is a potential role for $\mathrm{r}-\mathrm{HLH}$ in this population

Fig. 4: Change in luteinizing hormone receptors of ovaries with advancing age to add-back LH will depend on clinical tests and equipment available. The monitoring of follicular progression, estradiol concentrations and endometrial thickness as a clinical measure of LH response is suggested. ${ }^{14,15}$

\section{FSH/LH RATIO}

Day-3 gonadotropin and estradiol testing is typically included in the evaluation of ovarian reserve and the prediction of response to ovarian stimulation. Several authors have proposed utilizing the basal FSH:LH ratio as a predictor of ovarian response and ART success. Some did not find any differences in outcomes between patients with an FSH:LH ratio $>3: 1$ vs those $<3: 1$. This is in contrast to several other retrospective studies which have shown significantly better outcomes in patients with an FSH:LH ratio <3:1, with more oocytes retrieved, more metaphase-II oocytes, higher fertilization rates and increased pregnancy rates. Orvieto et al retrospectively evaluated patients with FSH:LH ratios of $>2: 1$ and $>3: 1$ who had undergone treatment with two assisted cycles, once with an HMG-only protocol and once with $\mathrm{rFSH}-$ only protocol. When the HMG cycles were compared with $\mathrm{rFSH}$ cycles, the HMG cycles resulted in significantly higher peak estradiol, a higher oocyte maturity rate, better embryos, a higher implantation rate and a higher clinical pregnancy rate. However, these studies are retrospective and there is no randomized controlled data to demonstrate if the use of LH is beneficial for patients with an elevated FSH:LH ratio. More research is required to determine if precycle testing can yield predictors that identify patients most likely to benefit from additional exogenous $\mathrm{LH}^{36}$

\section{POLYCYSTIC OVARY SYNDROME}

The detrimental impact of this endocrinological disorder is linked to hypersecretion of LH and ovulatory dysfunction. Increased LH with elevated LH:FSH ratio is a characteristic of PCOS. Waldstreicher et al demonstrated that women with PCOS have persistently rapid LH (GnRH) pulse frequency, in the order of one pulse per hour, without the normal cyclic variation seen in ovulatory women. The persistently rapid LH pulsatility, characteristic of PCOS probably signifies a failure of the systems necessary to suppress GnRH pulsatility, rather than representing an acceleration of the GnRH pulse generator. The mRNA expression of $\mathrm{LH}$ receptor is excessive in Granulosa cells of antral follicles causing premature action of LH in PCOS. Temporary normalization occurs in the late luteal and early follicular phase before progesterone induced bleeding. These are some of the proposed explanations though the exact role of LH in PCOS is yet to be confirmed. 
Studies have found that such women are associated with poor fertilization, oocyte quality and embryo quality, which could be due to underlying mechanisms, such as androgen excess induced by LH. However, contrary to previous belief, it is now clear that hyperinsulinemia and not LH hypersecretion plays a vital role in polycystic ovary syndrome (PCOS) pathogenesis. Adding LH in this scenario would lead to OHSS and hence LH should be avoided.$^{37}$ Studies have also demonstrated that in patients with polycystic ovary syndrome, supplementation with $225 \mathrm{IU}$ or $450 \mathrm{IU}$ rLH in the late phase decreased the number of follicles on the day of HCG administration compared with placebo. ${ }^{38}$

Thus, the role of LH in PCOS is still not clearly understood. In practice, individual patient response should be assessed and LH may be added in hyporesponders or severely down regulated patients with caution. There may be a role of exogenous LH in late follicular phase in a select group of steady responders.

\section{EXOGENOUS LH SUPPLEMENTATION IN ASIAN POPULATION}

There are wide variations in ovarian response in patients so the treatment regimes tailored to individual patient phenotypes and based on sound paracrine principles are more likely to achieve positive outcomes. Asian assisted reproduction practitioners make use of both long agonist and antagonist protocols for ovarian stimulation. Published literature on the beneficial effects of exogenous LH in patients with previous suboptimal response or low baseline serum LH concentrations is more extensive in long agonist protocols. ${ }^{20,34}$

The recommendations based on literature review and expert opinion regarding exogenous LH apply mainly to the use of GnRH agonists. The evidence for addition of rLH to r-HFSH in antagonist protocols for ovarian stimulation is inconclusive and future studies are awaited (Table 1$)^{18}$

\section{DOSE AND TIMING OF INITIATION OF r-HLH}

The dose of r-HLH in hypogonadotropic hypogonadism patients as stated in the summary of product characteristics for r-HLH is $75 \mathrm{IU}$ combined with $150 \mathrm{IU}$ of r-HFSH, i.e. a 2:1 ratio of FSH to LH. In patients undergoing assisted reproduction technology with prevention of $\mathrm{LH}$ surge using GnRH analogs, most of the published studies on the combination of rLH and r-HFSH in suboptimal responders used rLH doses of 75 to 150 IU daily combined with r-HFSH doses of 300 to $375 \mathrm{IU}$. While this dose is sufficient in hypogonadotropic patients, it is unclear if higher doses are optimal in assisted reproduction patients. The studies of normal assisted reproduction populations have failed to clearly demonstrate the ideal LH dose.

Studies suggest that a small percentage of women may require up to $225 \mathrm{IU}$ of $\mathrm{rLH} /$ day subcutaneously, but high dose of rLH is also found to be immunogenic and well tolerated. To achieve an optimal benefit, Ramaraju et al also suggest a dose of 75 IU/day of rLH for supplementation with r-hFSH. ${ }^{35}$

The widely used dosage is a ratio of 2:1 for FSH:LH, i.e. 150 IU: 75 IU starting on day 1 or days 6 of stimulation, especially in hypogonadotropic hypogonadism patients and is an appropriate dose to drive follicular development, estradiol production and endometrial development. A study showed that the administration of $\mathrm{rLH}$ (75 IU/day for 4 days), 1 day before the beginning r-HFSH stimulation, offers some benefits in terms of clinical pregnancies when compared with the patients undergoing stimulation with r-HFSH alone. ${ }^{18}$

The timing of initiation of rLH in ovarian stimulation is inconsistent in practice. In some protocols, patients start on rLH from day 1 of stimulation and for others patients start on days 6 to 8 . Currently, there is no evidence supporting either day 1 or days 6 to 8 for starting rLH. However, theoretically, there may be a benefit to start patients on day 1 if a clinician wants to maximize the benefit of increased ovarian androgen production even though the cost may increase. LH supplementation from day 1 may increase circulating androgen concentrations, which in combination with FSH can act synergistically to promote FSH receptor mRNA expression, follicular development and steroidogenesis. ${ }^{16-18}$

Studies have also fortified that rLH in combination with FSH is better than HMG with FSH. This might be due to excessive or inconsistent LH activity from the hCG

Table 1: Consensus on recommended use of luteinizing hormone in Asian women undergoing long gonadotropin-releasing hormone agonist protocols

\begin{tabular}{ll}
\hline Patient category & Indication \\
\hline $\begin{array}{l}\text { Substantial evidence of } \\
\text { benefit of r-LH in addition to } \\
\text { r-FSH }\end{array}$ & $\begin{array}{l}\text { Known poor responders (oocyte } \\
\text { count }<4 \text { in previous cycle) }\end{array}$ \\
& $\begin{array}{l}\text { Midfollicular (days 6) suboptimal } \\
\text { response on long agonist }\end{array}$ \\
& $\begin{array}{l}\text { 1. No follicles }>10 \mathrm{~mm} \\
\text { 2. Estradiol }<200 \mathrm{pg} / \mathrm{ml}\end{array}$ \\
& $\begin{array}{l}\text { 3. Endometrial thickness }<6 \mathrm{~mm} \\
\text { Age }>35 \text { years started on }\end{array}$ \\
Some evidence of benefit of & $\begin{array}{l}\text { ovarian stimulation with either } \\
\text { the long agonist or antagonist }\end{array}$ \\
& protocol \\
Further research needed to & Biomarkers, e.g. variant LH \\
determine benefit of r-LH & \\
& Low baseline serum LH $<1.2 \mathrm{IU} / \mathrm{I}$ \\
& Low antral follicle count \\
& Low anti-Mullerian hormone \\
\hline
\end{tabular}


component in HMG that may affect oocyte maturation in the latter half of the ovarian stimulation cycle, giving rise to the differences in numbers of oocytes retrieved and success of pregnancy.

Concluding from the accumulated data, $75 \mathrm{IU}$ LH is sufficient to develop follicles, estradiol and endometrial growth in even the most profoundly LH-suppressed patients. Further studies are required to determine if higher doses are beneficial or detrimental to follicular development and the endometrium.

\section{CONCLUSION}

The synergistic yet complex LH and FSH interactions are imperative for adequate follicular development, ovulation and endometrial advancement. According to the LH threshold concept, there is a minimum and maximum effective dose for LH. The LH therapeutic window is best observed in two patient groups, namely hypogonadotropic hypogonadism patients or LH polymorphism and patients who are profoundly suppressed by downregulation with either $\mathrm{GnRH}$ agonists or antagonists in assisted reproduction technology. It is now recognized that with GnRH analog protocols in ovarian stimulation, levels of LH bioactivity in some patients (e.g. age >35) may be reduced to below the threshold and they may benefit from adjuvant rLH.

The best predictive factor for need of exogenous $\mathrm{LH}$ in assisted reproduction technology is a prior poor or suboptimal response to ovarian stimulation. There is increasing evidence that age is an important marker of deficient LH bioactivity in women undergoing assisted reproduction technology, with multiple studies showing benefit in women aged above 35 years. Another patient demographic that may benefit from adjuvant rLH in addition to r-HFSH are poor responders and women who exhibit suboptimal ovarian response during ovarian stimulation. These recommendations mostly apply to $\mathrm{GnRH}$ agonist protocols where there is substantial evidence for the use of adjuvant rLH.

Current evidence supports the use of HMG to improve outcomes in long GnRH agonist cycles. Data on using LH or HMG in GnRH antagonist cycles is currently limited, but substantial biological plausibility exists for LH supplement use in these cycles. This is due to the profound and rapid LH suppression that occurs at a time in follicle development dominated by LH activity. There is insufficient evidence to make definitive conclusions on the need for exogenous LH activity in GnRH antagonist cycles or the benefit of recombinant LH and hCG protocols. The use of both rLH and hCG to supplement ovarian stimulation may be associated with a substantial increase in stimulation cost and therefore more studies are needed to establish benefit both in terms of outcomes and cost effectiveness.

The research in hypogonadotropic patients suggests that $75 \mathrm{IU}$ of LH has demonstrable biological activity. However, studies have not established an optimal dose of LH or an optimal ratio of FSH:LH supplementation to maximize assisted reproduction outcomes. It is possible that such doses or ratios are patient specific and should be adjusted based on patient response to stimulation. Further research is indicated for development of variable FSH: LH ratio in drugs. In conclusion, the importance of LH in the biological process of follicular development and oocyte maturation should not be forgotten. Biomarkers to ascertain women who are in need of exogenous LH need to be sought. Future research should focus on the benefits and cost effectiveness of rLH and hCG; and methods for identifying patients who are most likely to benefit from LH supplementation, either with prestimulation or intrastimulation parameters.

\section{SUMMARY}

There is a dilemma regarding the usefulness of LH supplementation in controlled ovarian stimulation despite growing understanding of the LH and FSH interrelation and their effects on fertilization and implantation. The use of recombinant human LH in controlled ovarian hyperstimulation should be guided by a rationale that is based on the individualized patient profile. The use of LH has been recommended in older patients ( $>35$ years) as about 15 to $20 \%$ of women have less sensitive ovaries with increasing age. Poor responders, those with deeply suppressed endogenous LH like hypogonadotropic hypogonadism and patients with LH suppression during ART benefit from rLH supplementation. Hyporesponders where FSH and AFC is considered adequate at the start of the stimulation cycle seem to respond better with the addition of rLH. Single nucleotide polymorphisms of LH-receptors should be considered in such patients. Further research is needed to identify adequate dosing, cost efficacy, need for rLH and hCG supplementation in different patient profiles for maximum benefit during COS.

\section{REFERENCES}

1. Jeppesen JV, Kristensen SG, Nielsen ME, et al. LHR gene expression in human granulosa and cumulus cells from antrel and pre ovulatory follicles. J Clin Endocrinol Metab 2012b Aug;97:E1524-E1531.

2. McGee EA, Hsueh AJ. Initial and cyclic recruitment of ovarian follicles. Endocr Rev 2000 Apr;21(2):200-214. 
3. Chappel SC, Howles C. Reevaluation of the roles of luteinizing hormone and follicle stimulating hormone in the ovulatory process. Hum Reprod 1991;6(9):1206-1212.

4. Balasch J, Fábregues F. Is luteinizing hormone needed for optimal ovulation induction? Curr Opin Obest Gyncol 2002; 14(3):265-274

5. Hillier SG. Gonadotropic control of ovarian follicular growth and development. Mol Cell Endocrinol 2001 Jan;179(1-2):39-46.

6. Shoham Z. The clinical therapeutic window for luteinizing hormone in controlled ovarian stimulation. Fertil Steril 2002; 77(6):1170-1177.

7. Shemesh M. Actions of gonadotrophins on the uterus. Reproduction 2001 Jun;121(6):835-842.

8. Melmed S, Polonsky KS, Larsen PR, HMK, editors. Williams Textbook of Endocrinology: 12th ed. Philadelphia, PA: Saunders Elsevier; 2011.

9. O'dea L, O'brien F, Currie K, et al. Follicular development induced by recombinant luteinizing hormone (LH) and follicle-stimulating hormone (FSH) in anovulatory women with LH and FSH deficiency: evidence of a threshold effect. Curr Med Res Opin 2008 Oct;24(10):2785-2793.

10. Filicori M. Use of luteinizing hormone in the treatment of infertility: time for reassessment? American Society for Reproductive Medicine. Fertil Steril 2003 Feb;79(2):253-255.

11. van de Weijer BH, Mulders JW, Bos ES, et al. Compositional analyses of a human menopausal gonadotrophin preparation extracted from urine (menotropin). Identification of some of its major impurities. Reprod Biomed Online 2003 Nov;7(5): 547-557.

12. Al-Inany HG, Abou-Setta AM, Aboulghar MA, et al. Efficacy and safety of human menopausal gonadotrophins versus meta-analysis recombinant FSH: a meta-analysis. Reprod BioMed Online 2008 Jan;16(1):81-88.

13. Coomarasamy A, Afnan M, Cheema D, et al. Urinary HMG versus recombinant $\mathrm{FSH}$ for controlled ovarian hyperstimulation following an agonist long down-regulation protocol in IVF or ICSI treatment: a systematic review and meta-analysis. Hum Reprod 2008 Feb;23(2):310-315.

14. Bosch E, Labarta E, Crespo J, et al. Impact of luteinizing hormone administration on gonadotropin-releasing hormone antagonist cycles: an age-adjusted analysis. Fertil Steril 2011 Mar;95(3):1031-1036.

15. Hill MJ, Levens ED, Levy G, et al. The use of recombinant luteinizing hormone in patients undergoing assisted reproductive techniques with advanced reproductive age: a systematic review and meta-analysis. Fertil Steril 2012 May; 97(5):1108-1114.

16. The European Recombinant Human LH Study Group. Recombinant human luteinizing hormone (LH) to support recombinant human follicle-stimulating hormone (FSH) induced follicular development in LH- and FSH-deficient anovulatory women: a dose-finding study. J Clin Endocrinol Metab 1998;83(5):1507-1514.

17. Ferraretti AP, Gianaroli L, Magli MC, et al. Exogenous luteinizing hormone in controlled ovarian hyperstimulation for assisted reproduction techniques. Fertil Steril 2004;82(6): 1521-1526.

18. Wong PC, Qiao J, Ho C, et al. Current opinion on use of luteinizing hormone supplementation in assisted reproduction therapy: an Asian perspective. Reprod Biomed Online 2011;23(1):81-90.

19. Ruvolo G, Bosco L, Pane A, et al. Lower apoptosis rate in human cumulus cells after administration of recombinant luteinizing hormone to women undergoing ovarian stimulation for in vitro fertilization procedures. Fertil Steril 2007; 87(3):542-546.

20. Pezzuto A, Ferrari B, Coppola F, et al. LH supplementation in downregulated women undergoing assisted reproduction with baseline low serum LH levels. Gynecol Endocrinol 2010; 26(2):118-124.

21. Shoham Z, Smith H, Yeko T, et al. Recombinant LH (lutropin alfa) for the treatment of hypogonadotrophic women with profound LH deficiency: a randomized, double-blind, placebo-controlled, proof-of-efficacy study. Clin Endocrinol 2008 Sep;69(3):471-478.

22. Andersen AN. Lack of association between endogenous $\mathrm{LH}$ and pregnancy in GnRH antagonist protocols. Reprod Biomed Online 2011 Dec;23(6):692-694.

23. Grondahl ML, Borup R, Lee $Y B$, et al. Differences in gene expression of granulosa cells from women undergoing controlled ovarian hyperstimulation with either recombinant follicle-stimulating hormone or highly purified human menopausal gonadotropin. Fertil Steril 2009 May;91(5): 1820-1830.

24. Hugues JN, Soussis J, Calderon I, et al. On behalf of the recombinant LH study group. Does the addition of recombinant LH in WHO group II anovulatory women over-responding to FSH treatment reduce the number of developing follicles? A dose-finding study. Hum Reprod 2005 Mar;20(3):629-635.

25. Filicori M, Cognigni GE, Gamberini E, et al. Efficacy of low-dose human chorionic gonadotropin alone to complete controlled ovarian stimulation. Fertil Steril 2005 Aug;84(2): 394-401.

26. Baruffi R, Mauri AL, Petersen C, et al. Recombinant LH supplementation to recombinant FSH during induced ovarian stimulation in the GnRH-antagonist protocol: a metaanalysis. Reprod Bio Med Online 2007 Jan;14(1):14-25.

27. Kolibianakis EM, Kalogeropoulou L, Griesinger G, et al. Among patients treated with FSH and GnRH analogues for in vitro fertilization, is the addition of recombinant $\mathrm{LH}$ associated with the probability of live birth? A systematic review and meta-analysis. Hum Reprod Update 2007 SepOct;13(5):445-452.

28. Alviggi C, Clarizia R, Pettersson $\mathrm{K}$, et al. Suboptimal response to GnRHa long protocol is associated with a common LH polymorphism. Reprod Biomed Online 2009 Jan;18(1):9-14.

29. Mafra FA, Bianco B, Christofolini DM, et al. Luteinizing hormone beta-subunit gene (LH beta) polymorphism in infertility and endometriosis-associated infertility. Eur J Obstet Gynecol Reprod Biol 2010;151(1):66-69.

30. Humaidan P, Bungum L, Bungum M, et al. Ovarian response and pregnancy outcome related to mid-follicular LH levels in women undergoing assisted reproduction with GnRH agonist down-regulation and recombinant FSH stimulation. Hum Reprod 2002;17(8):2016-2021.

31. Acevedo B, Sanchez M, Gomez JL, et al. Luteinizing hormone supplementation increases pregnancy rates in gonadotropinreleasing hormone antagonist donor cycles. Fertil Steril 2004 Aug;82(2):343-347.

32. Hill MJ, Levy G, Levens ED. Does exogenous LH in ovarian stimulation improve assisted reproduction success? An appraisal of the literature. Reprod Biomed Online 2012; 24(3):261-271.

33. Bosdou JK, Venetis CA, Kolibianakis EM, et al. The use of androgens or androgen-modulating agents in poor 
responders undergoing in vitro fertilization: a systematic review and meta-analysis. Hum Reprod Update 2012 MarApr;18(2):127-145.

34. De Placido G, Alviggi C, Perino A, et al. Italian collaborative group on recombinant human luteinizing hormone. Recombinant human LH supplementation versus recombinant human FSH (rFSH) step-up protocol during controlled ovarian stimulation in normogonadotropic women with initial inadequate ovarian response to $\mathrm{rFSH}$. A multicentre, prospective, randomized controlled trial. Hum Reprod 2005; 20(2):390-396.

35. Raju GA, Teng SC, Kavitha P, et al. Combination of recombinant follicle stimulating hormone with human menopausal gonadotrophin or recombinant luteinizing hormone in a long gonadotrophin-releasing hormone agonist protocol: a retrospective study. Reprod Med Biol 2012;11(3):129-133.

36. Orvieto R, Meltzer S, Rabinson J, et al. Does day 3 luteinizinghormone level predict IVF success in patients undergoing controlled ovarian stimulation with GnRH analogues? Fertil Steril 2008 Oct;90(4):1297-1300.

37. Rekha PS. Is LH necessary in ovulation induction? In: Desai S, Parihar M, Allahabadia G, editors. Infertility: principles and practice. India: BI Publications Pvt Ltd; 2004. p. 5.

38. Loumaye E, Engrand P, Shoham Z, et al. Clinical evidence for an LH ceiling effect induced by administration of recombinant human LH during the late follicular phase of stimulated cycles in World Health Organization type I and type II anovulation. Hum Reprod 2003;18(2):314-322. 